\title{
Internações por Lúpus no estado de Alagoas: 2002-2007
}

\author{
Lupus admissions in the state of Alagoas: \\ 2002-2007
}

\author{
Ana Carolina Santos Silva1, Edla Cavalcanti Amorim', \\ Gabriela Gama Silva1, Waneska Alexandra Alves², \\ Divanise Suruagy Correia ${ }^{3}$
}

\begin{abstract}
Silva ACS, Amorim EC, Silva GG, Alves WA, Correia DS. Internações por Lúpus no estado de Alagoas: 2002-2007. Rev Med (São Paulo). 2010 jan.-mar.;89(1):43-9.

RESUMO: Objetivou-se identificar o número de internações por Lúpus no estado de Alagoas no período de 2002 a 2007 com a análise de alguns aspectos relevantes. O Banco de Dados do Sistema Único de Saúde (DATASUS) foi utilizado como fonte de dados. As variáveis abordadas foram retiradas da Ficha de Autorização Hospitalar (AIH) e analisadas no programa EPI INFO 3.5.1 através de medidas estatísticas de frequência. Nesse período, 578 pacientes com Lúpus foram internados no estado de Alagoas, prevalecendo o sexo feminino $(90,3 \%)$ e a média de idade de 24,36 anos. Quanto à procedência dos pacientes com Lúpus, a maioria era da mesorregião Leste, tendo destaque a cidade de Maceió (capital do estado), origem de $50,71 \%$ dos pacientes. A capital possuiu também o maior número de internações $(94,3 \%)$. A especialidade que realizou maior quantidade de diagnóstico principal e admissão de pacientes com Lúpus foi a Clínica Médica $(92,05 \%)$, sendo todas essas informações compatíveis com estudos semelhantes realizados em outros estados brasileiros. O mês de maior número de internações foi março, e o período de permanência de internação dos pacientes teve média de 7,24 dias. O custo médio por paciente foi de $R \$ 121,77$; por dia de internação de $R \$ 16,80$; e por ano de $\mathrm{R} \$ 115,18$, sendo esses dados divergentes daqueles encontrados na literatura, que mostra um custo em se manter o paciente com Lúpus por ano no Brasil de $R \$ 999,00$, valor significativamente superior. Foram a óbito $14(2,4 \%)$ dos pacientes estudados. Houve prevalência do diagnóstico principal de Lúpus Eritematoso Disseminado não especificado $(45,33 \%)$
\end{abstract}

DESCRITORES: Lúpus eritematoso sistêmico/epidemiologia; Hospitalização/estatística \& dados numéricos.

\footnotetext{
${ }^{1}$ Acadêmicas de Medicina. Faculdade de Medicina (FAMED), Universidade Federal de Alagoas (UFAL). ${ }^{2}$ Prof ${ }^{a}$ Ms., Faculdade de Medicina (FAMED), Universidade Federal de Alagoas (UFAL).

${ }^{3}$ Prof ${ }^{-}$Dr ${ }^{\text {a }}$, Faculdade de Medicina (FAMED), Universidade Federal de Alagoas (UFAL).

Endereço para correspondência: Gabriela Gama Silva. Av. Betel, 29, quadra G, Tabuleiro dos Martins, Maceió-AL. CEP: 57082-650. E-mail: g_gama_@ hotmail.com
} 


\section{INTRODUÇÃO}

$\mathrm{O}$ Lúpus é uma doença crônica auto-imune que se caracteriza pela produção de auto-anticorpos por linfócitos B modificados ${ }^{1}$. De etiologia ainda não propriamente definida, porém relacionada à interligação entre fatores ambientais e genes suscetíveis à doença, acomete mais mulheres, em idade reprodutiva, do que homens, apresentando a incidência de uma pessoa a cada 1.000 da raça branca, e uma em cada 250 pessoas negras ${ }^{2}$. Além disso, o Lúpus apresenta diversas complicações clínicas, principalmente artralgias, problemas renais e cardiopulmonare ${ }^{1}$, podendo afetar também o sistema nervoso central (SNC), levando à sintomatologia neurológica de convulsões, cefaléia e psicose².

Essa patologia pode apresentar-se sob diferentes formas: Lúpus Eritematoso Cutâneo, que tem a pele como órgão alvo e pode se classificar em agudo (Lúpus Eritematoso sistêmico com lesões cutâneas), subagudo e crônico (que é o Lúpus Eritematoso Discóide); Lúpus Eritematoso Sistêmico (LES), que se caracteriza por um transtorno multissistêmico marcado pelo acometimento da pele, articulações, rins e membranas serosas; Lúpus induzido por drogas, que é definido como o LES idiopático relacionado à exposição contínua a fármacos (por mais de 30 dias), havendo, normalmente, resolução do quadro com a suspensão do medicamento desencadeante ${ }^{3}$.

Do mesmo modo que ocorre nos doentes crônicos, a internação dos pacientes com LES é bastante frequente. Tal constatação pode ser explicada pelo fato de que as manifestações dessa doença podem acarretar um quadro grave de incapacidade ao doente, oferecendo grande risco à vida, sendo o envolvimento renal o mais relacionado à morbidade e à mortalidade da patologia ${ }^{4}$. A gravidade do quadro do paciente com LES pode levá-lo a necessitar de internamento hospitalar e de medidas de suporte à vida, além de cuidados médicos ${ }^{5}$, assim como é verificado em outras doenças crônicas que possuem a causa da internação relacionada à agudização dos problemas crônicos ${ }^{6}$, correspondendo à maior demanda de serviços de saúde, respondendo por parte considerável dos gastos efetuados no setor ${ }^{7}$.

O acima exposto justifica o presente estudo, que visa a apresentar o número de internações por Lúpus no estado de Alagoas no período de 2002 a 2007, correlacionando-os às variáveis: sexo, idade, local de internação e procedência, subclassificações da doença, óbitos, período e custos de internações, estabelecendo uma comparação com os estudos encontrados na revisão realizada sobre essa patologia no Brasil.

\section{MATERIAIS E MÉTODOS}

Trata-se de um estudo quantitativo, descritivo, transversal, que utilizou dados secundários obtidos do Sistema Único de Saúde (DATASUS) sobre Lúpus para sua composição.

A partir do Banco de Dados pesquisado (DATASUS $)^{17}$, foram extraídas as informações necessárias para o alcance dos objetivos propostos, ou seja, aquelas referentes ao número de internações por Lúpus no estado de Alagoas, numa série de cinco anos (2002-2007). Com a finalidade de melhor entendimento em relação à distribuição epidemiológica da patologia no estado de Alagoas, os dados foram subdivididos de acordo com tópicos presentes na Ficha de Internação Hospitalar ${ }^{18}$.

A referida subdivisão dos dados foi realizada com base nas variáveis: faixa etária, sexo, local de origem e de internação dos pacientes, especialidade médica responsável pelo atendimento, distribuição mensal e anual dos internamentos, dias de permanência dos pacientes internados, custos diários, anuais e gasto médio por paciente no período de internação, número de óbitos após internamento e classificação do tipo de Lúpus.

A amostra utilizada na análise correspondeu a um total de 578 internações, constando de pacientes com Lúpus em suas mais variadas manifestações, oriundos de todo o estado de Alagoas, nos cinco anos estudados. A análise dos dados foi realizada com o auxílio do programa Excel, no qual os números referentes às variáveis levantadas foram calculados, e tabelas e gráficos foram produzidos, facilitando a visualização dos resultados obtidos para posterior discussão dos mesmos.

\section{RESULTADOS}

No período de 2002 a 2007, em Alagoas, foram admitidos para internamento, em 24 hospitais do estado, um total de 578 pacientes com Lúpus; desse total, 90,3\% correspondiam ao sexo feminino, e $9,7 \%$, ao sexo masculino. As mulheres internadas apresentaram idade média de 30 anos, apresentando maior número de casos na faixa etária dos 34 , ou seja, $5,74 \%$ do total de mulheres do estudo, e 24 anos, 5,17\%; enquanto os pacientes masculinos apresentaram média de idade de 24 anos, e maior porcentagem na faixa etária de 24 anos, 14,54\% (Gráfico 1). 
Gráfico 1. Distribuição por sexo e faixa etária do número de casos por Lúpus no estado de Alagoas no período de 20022007

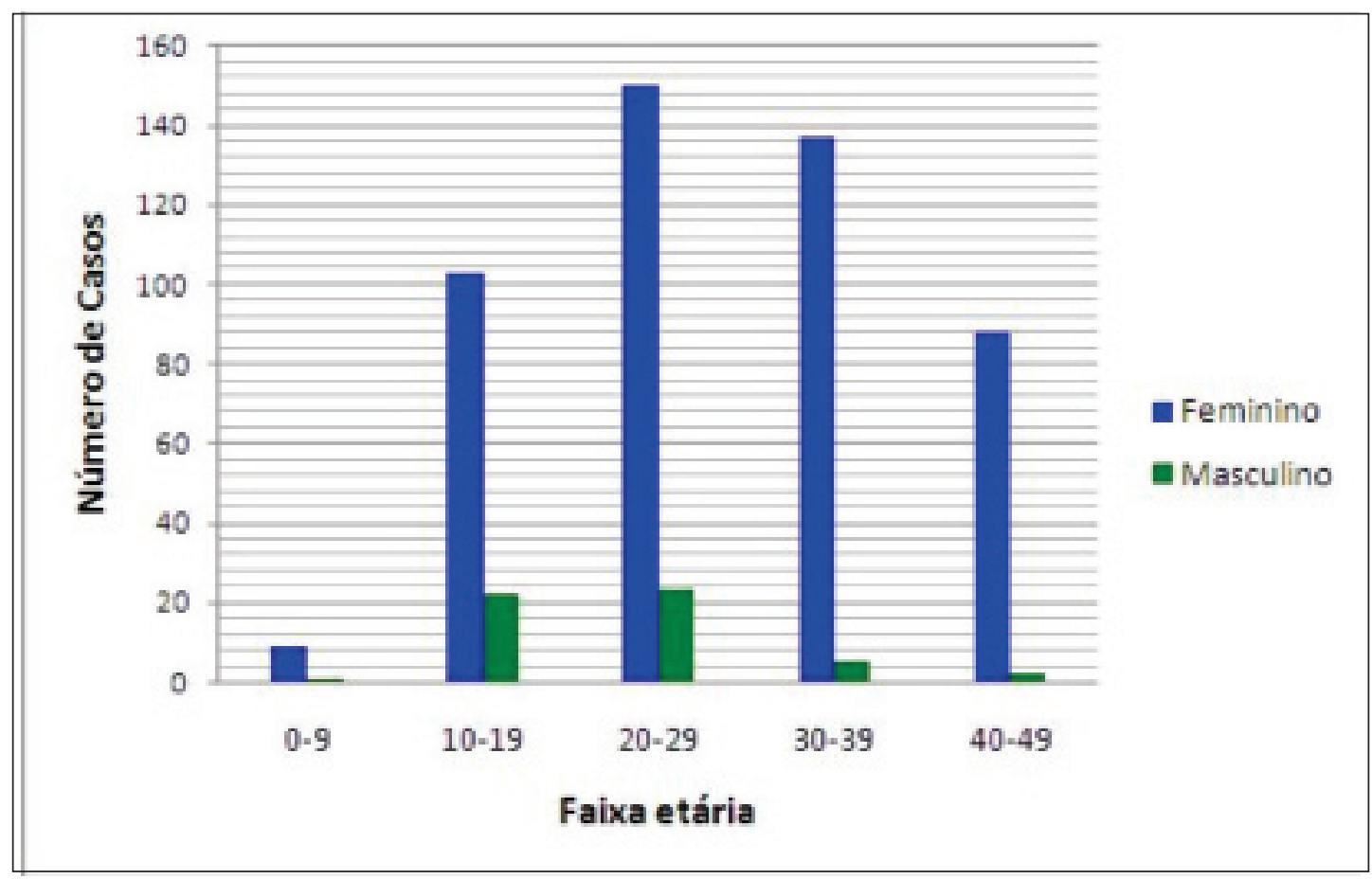

Os pacientes eram procedentes das três mesorregiões em que o estado é dividido, ou seja, $9 \%$ da mesorregião do Agreste, $12 \%$ da mesorregião do Sertão e a maior parcela, 79\%, da mesorregião do Leste (Tabela 1), onde se encontra a capital estadual. Considerando-se a distribuição por cidades, 50,17\% dos pacientes eram provenientes da capital do estado, isto é, Maceió; 3,97\% da cidade de Delmiro Gouveia, e 3,97\% do município de Arapiraca (Gráfico 2). Os demais $41,89 \%$ eram procedentes de outras cidades distribuídas pelas demais regiões do estado.
Tabela 1. Número de pacientes com Lúpus procedentes das três mesorregiões do estado de Alagoas no período de 2002-2007

\begin{tabular}{lcc} 
Mesorregião & N & $\%$ \\
\hline Leste & 455 & 78,72 \\
Sertão & 71 & 12,28 \\
Agreste & 52 & 9,00 \\
Total & 578 & 100 \\
\hline
\end{tabular}

Gráfico 2. Municípios com maior número de casos por Lúpus no estado de Alagoas no período de 2002-2007

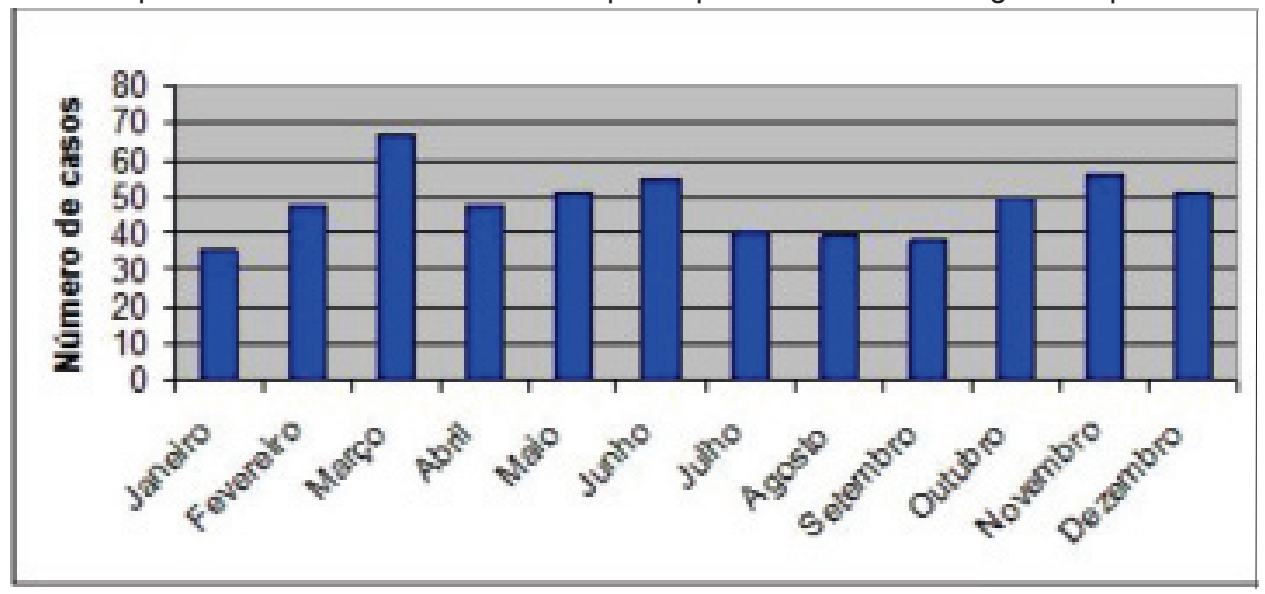


As internações ocorreram principalmente na capital Maceió - 94,3\% dos pacientes, no município de Pão de Açúcar - 1,5\%, em Arapiraca - 1,2\%, e em outras localidades $-3 \%$ dos pacientes. A especialidade médica responsável pelo diagnóstico principal e admissão dos pacientes para internação foi, em sua maioria, de Clínica Médica 92,05\%, sendo, secundariamente, a especialidade de
Pediatria 7,95\%.

$\mathrm{Na}$ distribuição anual, verificou-se um aumento do número de internações no período em estudo: de 76 pacientes em 2002, chegou-se a 135 pacientes em 2007. Mensalmente, houve uma flutuação no número de internações quantificadas, suspeitando-se de que seu pico tenha ocorrido no mês de março (Gráfico 3).

Gráfico 3. Número de internações por Lúpus por meses no estado de Alagoas no período de 2002-2007

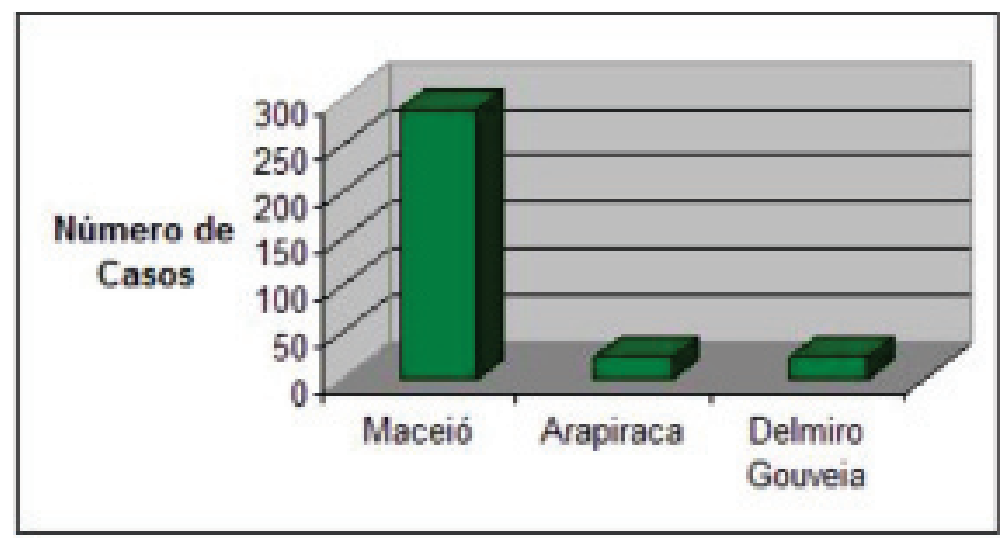

O período de permanência de internação dos pacientes teve a média de 7,24 dias por paciente, somando um total de 4.189 dias no intervalo de tempo estudado, havendo cerca de duas internações por dia. O valor total gasto durante todos os dias de internação corresponderam a $\mathrm{R} \$ 70.387,51$, verificando-se um custo médio por paciente de $\mathrm{R} \$ 121,77$; por dia de internação de $R \$ 16,80$; e por paciente por ano de $\mathrm{R} \$ 115,18$ (Tabela 2).

Tabela 2. Valores gastos com as internações realizadas em pacientes com Lúpus no estado de Alagoas no período de 2002-2007

\begin{tabular}{lc} 
& Valor $^{\star}$ \\
\hline Por dia de internação & 16,8 \\
Por paciente & 121,77 \\
Por paciente por ano & 115,18 \\
Total $^{* *}$ & $70.387,51$ \\
\hline
\end{tabular}

$\left(^{\star}\right)$ Valor calculado em reais; $\left(^{* *}\right)$ Total calculado no período de estudo 2002-2007.

Foram a óbito $14(2,4 \%)$ dos pacientes estudados. O diagnóstico principal identificado foi de Lúpus Eritematoso Disseminado não especificado, contabilizando-se $45,33 \%$ dos pacientes diagnosticados. Após, 21,28\% foram diagnosticados com Lúpus Eritematoso disseminado (outra forma), 5,89\% com Lúpus Eritematoso Disseminado comprometendo outros órgãos/sistema, 4,32\% com Lúpus Eritematoso Induzido por drogas, 20,42\% com Lúpus Eritematoso localizado, e 2,76\% com Lúpus Eritematoso Cutâneo subagudo (Tabela 3).
Tabela 3. Número de pacientes divididos por subclassificações da patologia Lúpus no estado de Alagoas no período de 2002-2007

\begin{tabular}{|c|c|c|}
\hline Diagnóstico principal & $\mathrm{N}$ & $\%$ \\
\hline $\begin{array}{l}\text { Lúpus Eritematoso Disseminado } \\
\text { não especificado }\end{array}$ & 262 & 45,33 \\
\hline $\begin{array}{l}\text { Lúpus Eritematoso Disseminado } \\
\text { (outra forma) }\end{array}$ & 123 & 21,28 \\
\hline Lúpus Eritematoso Localizado & 118 & 20,42 \\
\hline $\begin{array}{l}\text { Lúpus Eritematoso Disseminado } \\
\text { comprometendo outros órgãos/sistema }\end{array}$ & 34 & 5,88 \\
\hline Lúpus Eritematoso Induzido por drogas & 25 & 4,32 \\
\hline $\begin{array}{l}\text { Lúpus Eritematoso Cutâneo } \\
\text { subagudo }\end{array}$ & 16 & 2,77 \\
\hline Total & 578 & 100 \\
\hline
\end{tabular}

\section{DISCUSSÃO}

Não foram encontrados estudos epidemiológicos publicados relativos ao Lúpus no estado de Alagoas verificados na literatura. Em similitude aos demais estudos analisados, em que há uma incidência da doença na proporção de nove a dez mulheres para um homem ${ }^{8}$, o presente trabalho mostrou uma prevalência da doença no sexo feminino de $90,3 \%$, enquanto que a porcentagem de homens encontrados na amostra foi de $9,7 \%$. 
A doença sistêmica (LES) apresenta-se, frequentemente, em mulheres jovens (20-40 anos), crianças e neonatos $^{9}$; esse intervalo de idade condiz com o encontrado nesse estudo, que verificou um maior percentual de pacientes mulheres na faixa de 20-49 anos, com média de idade de 30 anos; por sua vez, os homens apresentaram média de 24 anos. Glezer et al. ${ }^{10}$ observou que a maioria das doenças autoimunes incide sobre as mulheres, e que seu agravamento durante a gestação, e no período periparto, sugere associação entre estrógeno, prolactina e a atividade imunológica, o que pode explicar a maior prevalência do Lúpus, enquadrado nessa classificação de doenças, nesse grupo, em relação ao identificado no sexo masculino.

Martinez et al. ${ }^{11}$ afirma que os possíveis fatores envolvidos na gênese do Lúpus incluem: componente genético; componentes constitucionais, como hormônios, e ambientais, como o sol, sendo o fenômeno da fotossensibilidade um aspecto central, de modo que os pacientes são orientados a se protegerem da exposição solar.

Assim, por se situar na região Nordeste do país, que possui um clima quente com altas temperaturas ao longo de todo o ano, a localização do estado de Alagoas por si só pode ser um fator contribuinte para o agravamento dessa patologia, sendo esse processo de gênese mais evidenciado na mesorregião Leste do estado - que possui a maior parcela da população - , seguida da mesorregião do Sertão, onde a incidência de raios ultravioleta é ainda superior à das demais regiões.

No período de 2002 a 2007, houve um aumento do número de internações por Lúpus no estado, fator que sugere a ampliação nas buscas por tratamento e suporte pelos doentes. A cidade de Maceió recebeu o maior número de internações, correspondendo a $94,3 \%$ do total, e também foi o município em que se pôde verificar o maior número de casos, $50,17 \%$, seguida pelas cidades sertanejas de Delmiro Gouveia e de Arapiraca, com apenas $3,97 \%$ dos casos, demonstrando, pois, a maior disponibilidade de recursos médicos oferecidos na capital alagoana e, por consequência, um déficit nas demais regiões do estado.

O consumo de internações hospitalares foi três vezes superior para doentes crônicos em comparação aos demais pesquisados em estudo realizado no Brasil em 19987, o que serve de parâmetro para o número encontrado de duas internações por dia e 689 por ano de Lúpus no período estudado.

O LES e sua terapêutica causam alterações no estilo de vida, e mesmo redução da capacidade funcional do indivíduo doente, que podem alterar de forma significativa a qualidade de vida do paciente ${ }^{11}$; do mesmo modo, entre as queixas mais comumente apresentadas pelos pacientes com LES, estão: fadiga, mal-estar, anorexia, perda ponderal e febre ${ }^{12}$; levando à morte do paciente quando não devidamente tratadas. Nesse estudo, os óbitos corresponderam a $2,4 \%$ dos casos analisados, número relativamente baixo se comparado à mortalidade apontada pela literatura estudada, de $16,2 \%$ de pacientes com LES de início na infância, e 11,7\% com LES de início na idade adulta, verificado em estudo realizado com 75 pacientes com LES cujo início da doença se deu antes dos 16 anos, e 393 pacientes com início anterior a essa data ${ }^{13}$.

Estudos realizados quanto ao custo de doenças no Brasil verificaram que o valor médio anual gasto por paciente com Lúpus Eritematoso Sistêmico corresponde a R $\$ 1.914,00$, e os custos diretos médicos, a $\mathrm{R} \$ 999,00^{14}$; enquanto em estudo realizado com 100 pacientes no ambulatório de Reumatologia da Escola Paulista de Medicina ${ }^{15}$, inferiu-se que esses custos correspondiam a $\mathrm{R} \$ 2.287,44$, o que foi superior ao apresentado no país. No presente estudo, verificou-se um gasto anual por paciente com Lúpus de $\mathrm{R} \$ 115,18$, e por dia de internação de $R \$ 16,80$. Esses números diferem dos demais descritos, salientando-se que maior ou menor custo em internações é dependente do grau de tecnologia empregada nos hospitais ${ }^{14,15}$. Não foram encontrados na revisão de literatura mais detalhes que embasassem outras discussões pertinentes.

As especialidades médicas que admitiram pacientes para internação por Lúpus corresponderam à Clínica Médica (92,05\%), e à Pediatria (7,95\%). Tal achado pode ser explicado pelo fato de ser a Clinica Médica a especialidade cujos médicos em exercício costumam apresentar uma visão generalista do paciente, facilitando o diagnóstico da patologia estudada ou de complicações oriundas da mesma. A idade de início dos sintomas está entre os 16 e 55 anos em aproximadamente $55 \%$ dos pacientes ${ }^{15}$, fato que explica o número mencionado de internações realizadas pela Pediatria.

O principal diagnóstico apresentado foi o de Lúpus Eritematoso Sistêmico, podendo: haver comprometimento de outros órgãos, ser caracterizado como não especificado ou apresentar-se de outra forma diferente das já citadas. Em contrapartida, menos de $3 \%$ dos diagnósticos eram de Lúpus Eritematoso Cutâneo Subagudo (LECSA) e, apesar do conhecimento de que a pele é acometida em $85 \%$ dos casos na doença sistêmica e de que o LECSA pode representar doença sistêmica em aproximadamente $50 \%$ dos casos ${ }^{17}$, essa pequena porcentagem de internações seria justificada pelo fato 
de que o acometimento cutâneo por si só é menos grave do que o sistêmico, causando assim menor número de internações em relação ao LES.

Considera-se que mais de $10 \%$ dos casos de LES são droga-induzidos ${ }^{3}$, podendo essa variável ser comparada aos 4,32\% contabilizados nessa pesquisa; e 20\% de diagnóstico de Lúpus Eritematoso Localizado, frisando-se que os dados obtidos são referentes ao número de internações e não necessariamente ao número específico de pacientes com Lúpus existentes no estado de Alagoas.

\section{CONCLUSÃO}

O número de internações por Lúpus no estado de Alagoas reitera dados encontrados nas referências bibliográficas pesquisadas, tais como: maior prevalência dessa patologia no sexo feminino e incidência maior na faixa etária de idade reprodutiva. Verificou-se também um crescimento do número de internações de pacientes com Lúpus no período estudado, maior procedência de pacientes de cidades de maior densidade populacional do estado, e reduzido número de pacientes que foram a óbito.

Agradecemos ao Profọ . Dr. Jairo Calado pela colaboração na obtenção dos dados.

Silva ACS, Amorim EC, Silva GG, Alves WA, Correia DS. Lupus admissions in the state of Alagoas: 2002 -2007. Rev Med (São Paulo). 2010 jan.-mar.;89(1):43-9.

\begin{abstract}
The objective of this study was to identify the number of hospitalizations for Lupus in the state of Alagoas during the period of 2002 to 2007 and to analyze its important aspects. The Health Information System (DATASUS) was used as source of referring data to the hospitalizations. The discussed variables were withdrawn from the Record Room from the Hospital Admission Authorization (AlH) and analyzed in the EP INFO program through statistical measurements of frequency. In this period, 578 lupus patients were admitted into hospitals in Alagoas State, $90.3 \%$ of whom were female with an average age of 24.36 years. The patients' provenience also was taken into account, and most of them came from the Eastern region of the state, especially from the city of Maceió, origin of $50.71 \%$ of the patients. This municipal district also held the majority of admissions (94.3\%). The Department of General Medicine was the one responsible for largest amount of main diagnosis and patients' admissions (92.05\%), all of these which were compatible with previous studies about Lupus in other places. March was the month with the largest number of hospitalizations (67). The average time of hospitalization was 7.24 days. The total value expenses during all days corresponded to $R \$ 70.387,51$, with an average cost of $R \$ 121,77$ per patient and a day cost of $R \$ 16,80$, being these data very divergent from those found in literature. Fourteen (2.4\%) of the studied patients died. The main diagnosis of Disseminated not specified Erythematous Lupus (45.33\%) prevailed.
\end{abstract}

KEY WORDS: Lupus erythematosus, systemic/epidemiology; Hospitalization/statistics \& numerical data.

\title{
REFERÊNCIAS
}

1. Peakman M, Vergani D. Imunologia: básica e clínica. Rio de Janeiro: Guanabara; 1999.

2. Ayache DCG, Costa IP. Alterações da personalidade no lúpus eritematoso sistêmico. Rev Bras Reumatol [online]. 2005,45(5):313-8.

3. Mota LMH, Haddad GP, Lima RAC, Carvalho JF, Muniz-Junqueira MI, Santos Neto LL, et al. Lúpus induzido por drogas: da imunologia básica à aplicada. Rev Bras Reumatol [online]. 2007,47(6):431-7.
4. Veloso VSP. Envolvimento renal em pacientes com Lúpus eritematoso sistêmico [dissertação]. Goiânia: Convênio Rede Centro-Oeste, Universidade de Brasília - UFG, UFMS; 2006.

5. Assis ECV, Oliveira JBB. Complicações pleuropulmonares no Lúpus eritematoso sistêmico: abordagem fisioterapêutica. Rev Bras Promoção Saúde [online]. 2004,17(1):92-6.

6. Martins LM, Franca APD, Kimura M. Qualidade de 
vida de pessoas com doença crônica. Rev Latinoam Enferm [online]. 1996, 4(3):5-18.

7. Almeida MF, Barata RB, Montero CV, Silva ZP. Prevalência de doenças crônicas auto-referidas e utilização de serviços de saúde, PNAD/1998, Brasil. Rev Ciên Saúde Coletiva [online]. 2002, 7(4):743-56.

8. Sociedade Brasileira de Reumatologia. Lúpus eritematoso sistêmico: acometimento cutâneo/articular. Rev Assoc Med Bras. 2006, 52(6):384-6.

9. Sampaio S, Rivitti E. Dermatologia. São Paulo: Artes Médicas; 2001.

10. Glezer A, Paraiba DB, Carvalho JF. O papel da prolactina no Lúpus eritematoso sistêmico: onde estamos. Rev Bras Reumatol [online]. 2009;49(2):153-7.

11. Martinez JE, Kitice AT, Airoldi DM, Giordano RH, Cadaval RAM. Avaliação do comprometimento ósseo no Lúpus eritematoso sistêmico. Rev Soc Bras Clin Med. 2008;6(2):49-52.

12. Bettero RG, Rahal MY, Barboza JS, Skare TL. Cefaléia no lupus eritematoso sistêmico: prevalência e condições associadas. Arq Neuropsiquiatr. 2007;65(4b):1196-9.

13. Costallat LTL, Appenzeller S, Marini R. Evolução e fatores prognósticos do lúpus eritematoso sistêmico em relação com a idade de início. Rev Bras Reumatol. 2002;42(2):91-8.

14. Onelli RM, Ferraz MB. Estudos de custos de doenças. Rev Bras Med. 2009;103-5.

15. Meinão IM. Utilização de recursos e custos em pacientes com Lúpus eritematoso sistêmico [dissertação]. Säo Paulo: Escola Paulista de Medicina, Universidade Federal de São Paulo; 2001.

16. Marini R. Contribuição ao estudo do Lúpus eritematoso sistêmico na infância: manifestações clínicas e laboratoriais de 59 pacientes [dissertação]. Campinas: Faculdade de Ciências Médicas, Universidade Estadual de Campinas; 1997.

17. Duarte AA. Lúpus eritematoso cutâneo. An Bras Dermatol (Rio de Janeiro). 2001;76(6):655-71.

18. Banco de dados do Sistema Único de Saúde (DATASUS). Disponível em: http://w3.datasus.gob. br/datasus/datasus.php.

19. Comunicação de internação hospitalar - manual de operação. Disponível em:http://www.saude.sc.gov. $\mathrm{br} / \mathrm{sih} /$ versoes/cih/PRESTADOR/Manual\%20de\%20 Operacao20CIH01.pdf. 